Article

\title{
Plug-in Electric Vehicles for Grid Services Provision: Proposing an Operational Characterization Procedure for V2G Systems
}

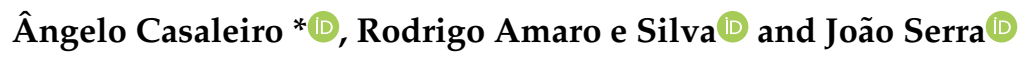 \\ Instituto Dom Luiz, Faculdade de Ciências da Universidade de Lisboa, Campo Grande 016, 1749-016 Lisboa, \\ Portugal; rasilva@fc.ul.pt (R.A.e.S.); jmserra@fc.ul.pt (J.S.) \\ * Correspondence: afcasaleiro@fc.ul.pt; Tel.: +351-914-139-599
}

Received: 17 February 2020; Accepted: 6 March 2020; Published: 7 March 2020

check for updates

\begin{abstract}
Plug-in electric vehicles (PEVs) are expected to play a role as power grid ancillary service providers through vehicle-to-grid (V2G) chargers, enabling higher levels of renewable electricity penetration. However, to fully exploit the storage capacity of PEVs and fast responsiveness, it is crucial to understand their operational characteristics. This work proposes a characterization procedure for $\mathrm{V} 2 \mathrm{G}$ systems providing grid services. It extends the existing literature on response time, AC/DC conversion and reactive power assessment. Illustrative results were obtained by implementing the procedure using a Nissan Leaf battery electric vehicle (BEV) connected to a remotely operated commercial V2G CHAdeMO charger. The V2G system was characterized as having a relative inaccuracy and variability of response inferior to $3 \%$ and $0.4 \%$, respectively. Its average communication and ramping times are $2.37 \mathrm{~s}$ and $0.26 \mathrm{~s} / \mathrm{kW}$, respectively. Its conversion efficiency and power factor both showed degradation in the power values below $50 \%$ of the charger's nominal power. Moreover, the proposed visualizations revealed that: i) the V2G system implements power requests for the DC power flow; ii) the power factor control algorithm was nonoperational; and iii) the acquired data can leverage statistical models that describe the operation of V2G systems (which is of extreme value for researchers and operators).
\end{abstract}

Keywords: ancillary services; CHAdeMO; PEV charger; test procedure

\section{Introduction}

Transportation electrification is expected to play a major role in the decarbonization of power systems, tackling climate change concerns and helping to achieve international carbon dioxide $\left(\mathrm{CO}_{2}\right)$ reduction commitments. However, it also poses several technical challenges to the operation of power grids, such as increased peak load, grid equipment overload and power quality degradation [1,2].

Unlike most electric loads, plug-in electric vehicles (PEVs) are equipped with batteries that can be charged at controllable power levels and scheduled time intervals. This allows for the design of charging strategies that mitigate the previously mentioned challenges and provide grid services [3], [4]. Vehicle-to-grid (V2G) enabled chargers extend this reality by allowing bidirectional power flows between PEVs and the power grid.

When aggregated, PEVs are capable of providing various grid services, such as power and energy balancing or grid contingencies [5-7]. Nonetheless, the fact that they can quickly shift from operating either as a load or power source, coupled with their fast ramping ability, makes PEVs-in a V2G context-more suited for primary and secondary reserve provision, where revenues can be maximized [8,9]. Reactive power compensation has also been proposed for V2G-enabled PEVs, which can be done simultaneously with active power transactions and without any additional impact to 
the battery state-of-charge (SOC) or lifetime [10,11]. Buja et al. (2017), however, pointed out some battery-related concerns related to electric vehicles (EVs) operated in V2G, either individually or as an aggregate, to provide such services [10]. These services can contribute to tackling the challenges in the distribution planning and operation of a power system introduced by increasing the penetration of distributed renewable electricity generation, reducing the need for substantial investments in the supply infrastructure expansion [9].

To fulfill the expected impacts of V2G in power grids, a detailed operational characterization of V2G systems is essential. It provides information which is of value for various V2G stakeholders and purposes: for operators, to fully explore PEVs in real-time grid service provisioning; for researchers, to leverage more realistic and, thus, more relevant modelling; and for regulators, to establish benchmarks and recommend best practices. The existing literature on this issue is scarce. Mouli et al. (2016) compared the response times of the CHAdeMO and Combined Charging System-Combo (CCS-Combo) fast charging protocols for V2G and dynamic charging [12]. The main difference between the two protocols is the type of communication used, with CHAdeMO and CCS-Combo making use of CAN (controller area network) bus communication and PLC (power line carrier) communication, respectively. In this work, the response times were measured as the time the charger takes to implement a sequence of requested power ramps. Martinenas et al. (2016) addressed the accuracy and response time for three locally controlled EVs in smart charging [13]. While the authors assumed an inaccuracy of less than 1 A in the current (around $200 \mathrm{~W}$ ), the response time is assessed as the duration of the ramping process. Additionally, the degree of correlation between the requested and effective signal seems to be suggested as an overall evaluation metric. Only Zecchino et al. (2019) proposed a more extensive and descriptive characterization procedure, as well as the assessment metrics (Table 1) which were implemented for a V2G CHAdeMO charger [14]. It requires both local and remote testing to distinguish between the time the equipment takes to react to a given order (named as the "starting time") and to effectively change its power output ("ramp-up/-down time"). Moreover, it assumes that ramping time is proportional to the requested ramping. Andersen et al. (2018) implemented this same procedure to test and compared various charger-EV combinations, showing that the combination (and not the charger alone) impacts the performance of a V2G system [5].

Table 1. Assessment metrics for vehicle-to-grid (V2G) systems as proposed in [14]. The presented information is derived from Table 2 in said reference, as well as from other sections of the text.

\begin{tabular}{lll}
\hline \multicolumn{1}{c}{ Metric } & \multicolumn{1}{c}{ Description } & \multicolumn{1}{c}{ Measured as ... } \\
\hline Set point linearity & $\begin{array}{l}\text { Discreetness of the provided } \\
\text { (dis)charging power set point }\end{array}$ & $\begin{array}{l}\text { The difference in active power flow between } \\
\text { two consecutive timestamps while } \\
\text { progressively varying the requested power } \\
\text { with time }\end{array}$ \\
\hline Accuracy & $\begin{array}{l}\text { Difference between the requested } \\
\text { and the delivered response }\end{array}$ & $\begin{array}{l}\text { The average deviation between the requested } \\
\text { and provided power over a time window } \\
\text { where power output is expected to be stable }\end{array}$ \\
\hline Set point precision & $\begin{array}{l}\text { Variation of the delivered response } \\
\text { for a given set point }\end{array}$ & $\begin{array}{l}\text { The difference between the maximum and } \\
\text { minimum values of the provided power over a } \\
\text { time window where power output is expected } \\
\text { to be stable }\end{array}$ \\
\hline $\begin{array}{l}\text { Starting time and maximum (or } \\
\text { total) activation time }\end{array}$ & $\begin{array}{l}\text { Time period between receiving a } \\
\text { given power request and its } \\
\text { effective implementation }\end{array}$ & $\begin{array}{l}\text { The time lag which maximizes the correlation } \\
\text { between the requested and the delivered } \\
\text { response in local and remote operation, } \\
\text { respectively }\end{array}$ \\
\hline Ramp-up \& Ramp-down time & $\begin{array}{l}\text { Up/downwards time between } \\
\text { activation time and full-service } \\
\text { provision, and vice versa }\end{array}$ & $\begin{array}{l}\text { The effective power variation divided by the } \\
\text { time needed by the equipment after activation }\end{array}$ \\
\hline
\end{tabular}

The aim of this work is, thus, to propose an extensive V2G characterization procedure (consisting of an experimental setup, a test pattern and assessment metrics) that address some limitations in the existing literature. Its main contributions are as follows: i) a clearer nomenclature for the assessment 
metrics; ii) a test pattern which evaluates power ramping in more detail; iii) a method for measuring the response time of V2G systems using only a single test pattern in remote operation; iv) a visualization focused on AC/DC power measurements for better understanding V2G operation; and v) the inclusion of reactive power considerations in the characterization procedure.

The implementation and expected outputs of the proposed procedure are also exemplified for a Nissan Leaf BEV connected to a remotely operated commercial V2G CHAdeMO charger.

\section{Proposed V2G System Characterization Procedure}

\subsection{General Description of the V2G Experimental Setup}

A grid services-providing V2G system consists of a PEV and electric vehicle supply equipment (EVSE), which allows for a bidirectional power flow and a connection point with the power grid. Furthermore, the EVSE should be supported by a control software that communicates with the PEV and can regulate its operation. To implement the proposed procedure, active and reactive power flows must be measured or inferred. Measuring additional variables, such as current or voltage and their harmonic distortion content, can add value and allow a better understanding of the V2G system and its impact on the power grid.

\subsection{Proposed Test Pattern}

The test pattern proposed in this work, illustrated in Figure 1, extends the one proposed in Zecchino et al. (2019) [14]. The original pattern can be divided in two sections, where the first is designed to assess the EVSE granularity and the second to assess its response to high-magnitude ramps. The pattern here proposed is designed so that the assessment also considers a range of power ramping requests.

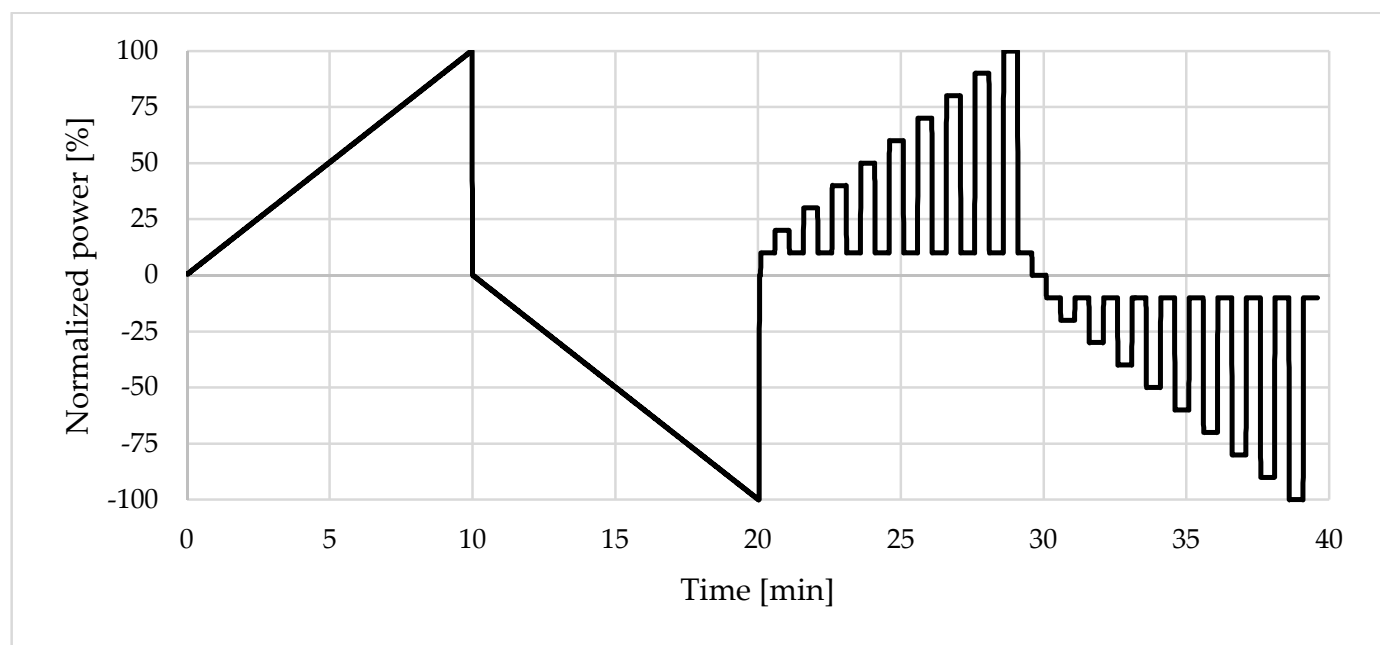

Figure 1. Proposed test pattern. Power requests are given to the electric vehicle supply equipment (EVSE), covering both charging and discharging operation modes and a range of power and power ramping values. A new power set point request is sent every $3 \mathrm{~s}$ during the first $20 \mathrm{~min}$, and $30 \mathrm{~s}$ for the remaining $20 \mathrm{~min}$.

Thus, in the first section (i.e., the first $20 \mathrm{~min}$ ), consecutive (i.e., separated by $3 \mathrm{~s}$ intervals) power set points are sent to the EVSE. The requests start at $0.5 \%$ of the EVSE's nominal power and progressively increase in $0.5 \%$ steps up to $100 \%$ (for the case study here considered, this corresponds to $50 \mathrm{~W}$ steps up to an output power of $10 \mathrm{~kW}$ ).

For the second section, a sequence of positive and negative power ramps with increasing magnitude, starting at $10 \%$ and increasing in $10 \%$ steps up to full nominal power (1 $\mathrm{kW}$ steps up to 10 
$\mathrm{kW}$ ). Each set point was separated by a $30 \mathrm{~s}$ interval to ensure stable measurements. Regarding sign convention, positive power denotes charging and negative power is discharging.

Each testing section should be implemented for both charging and discharging operation modes.

\subsection{Case Study Description}

In order to test the proposed procedure and obtain illustrative results, a Nissan Leaf 2015 model (the BEV), with a $24 \mathrm{kWh}$ lithium-ion battery, was connected to a commercial $10 \mathrm{~kW} \mathrm{CHAdeMO} \mathrm{V2G}$ outdoor charger (the EVSE). The EVSE, in turn, was connected to a customer electrical board. For continuous, real-time data acquisition (AC active and reactive power) a power and energy logger PEL 103 (Chauvin Arnoux Group, Paris, France) [15] was installed between the EVSE and the customer electrical board. The DC measurements were done using the EVSE internal DC current and voltage sensors, since the installation of external probes was not deemed suitable (as we used a site with public access). The complete experimental setup is illustrated in Figure 2.

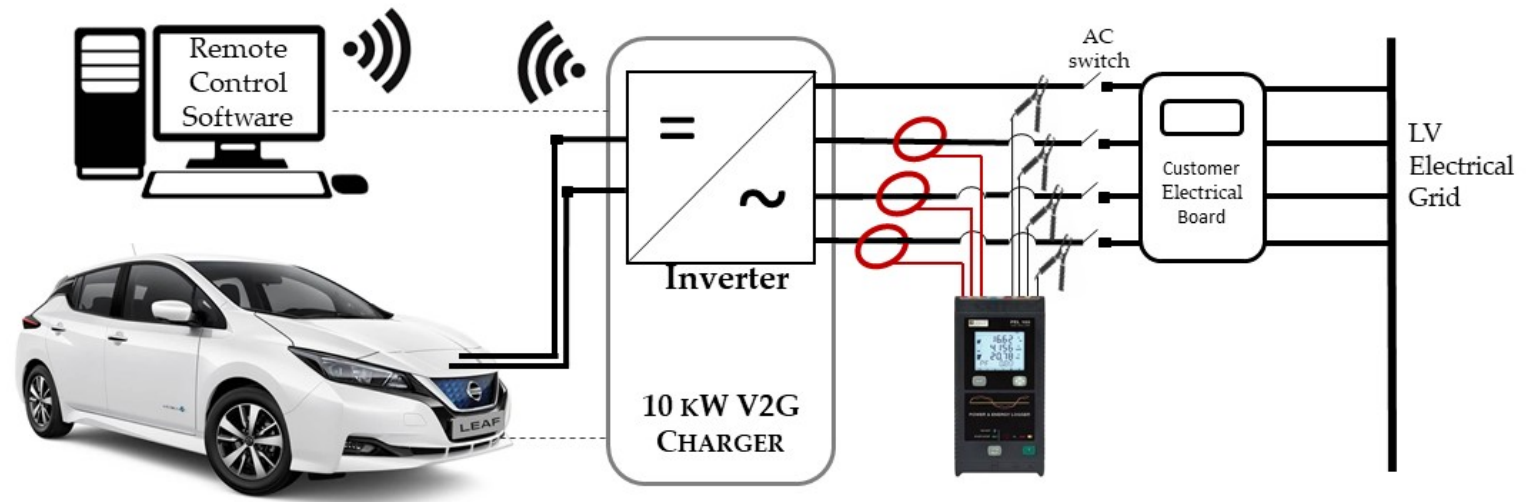

Figure 2. Experimental setup for the V2G system under analysis. The V2G system is remotely operated using a control software and measurements are done using a power and energy logger. Both power (continuous lines) and communication (dashed lines) flows are indicated.

The considered charger has five operation modes: i) "stop mode", where the equipment is in an idle state; ii) "simple charge", for PEVs that do not support V2G technology and, thus, can only be charged; iii) "V2G pause mode", where the equipment is in stand-by but ready for V2G operations; iv) "charge mode" and v) "discharge mode", where power flows from the grid to the PEV, or vice-versa, respectively. It is worth noting that the EVSE initiates at its nominal power $(10 \mathrm{~kW})$ and varies upon receiving power set points from the $\mathrm{V} 2 \mathrm{G}$ operator.

Additionally, and according to the standard IEC 61851-1 [16], this charger can be classified as Mode 4 (i.e., the PEV is indirectly connected to the AC supply network through an off-board charger, which is responsible for the AC/DC conversion) and the connection between the EVSE and the PEV is case $C$ (i.e., the power supply cable is permanently attached to the EVSE).

For remote operation, both $\mathrm{V} 2 \mathrm{G}$ systems have a built-in API based on proprietary communication protocols, allowing operators to send new power set points or to stop/pause its operation, as well as request its status (retrieving internal electrical measurements) at any given moment. Thus, the test pattern described in the previous section was implemented in Python 3.6 using said protocols.

\section{Metrics and Illustrative Results of a V2G System}

From the AC (active and reactive) and DC power flow measurements, various metrics can be calculated to characterize the V2G system. For example, Figure 3 shows the effective AC active power flow measured during the procedure. Measuring additional variables (e.g., current and voltage) can provide a better understanding of the results. 


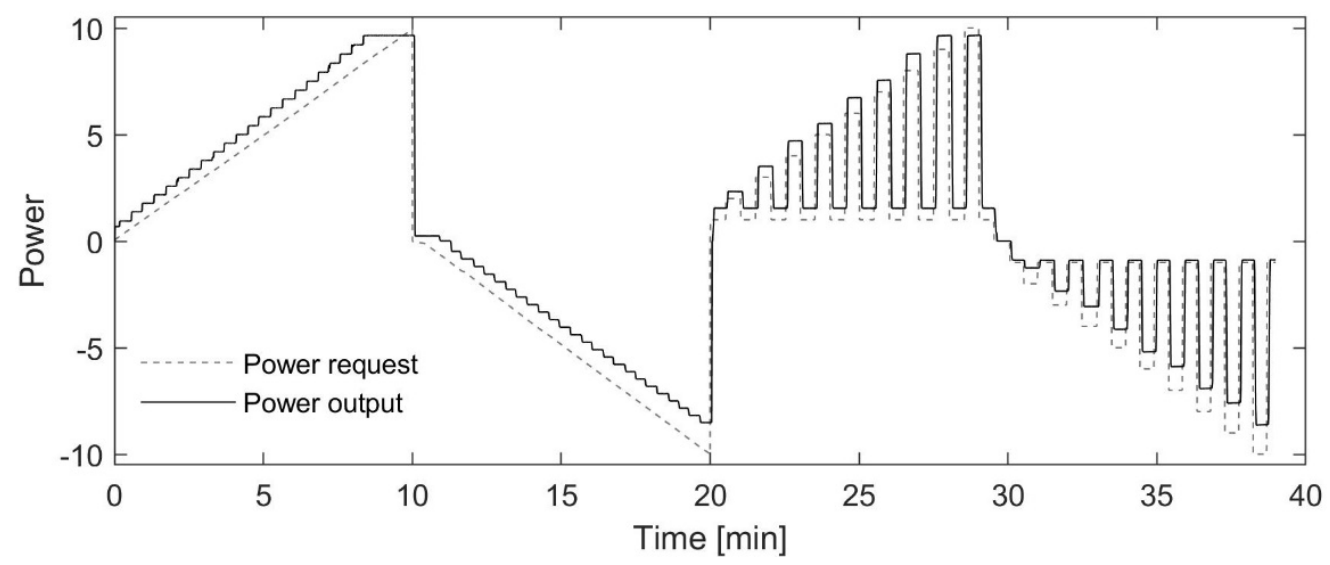

Figure 3. Power requests (in dashed grey) and effective AC active power flow (in black) during the implementation of the proposed procedure. Throughout the section, this data is used to calculate various characterization metrics for the tested V2G commercial charger.

\subsection{AC Power Granularity}

The power output granularity quantifies the level of discretization an EVSE is able to implement. Considering that the V2G concept is mainly proposed for grid services provision, this assessment should only focus on the AC power flow.

According to the CHAdeMO protocol [17], the EVSE can only implement integer DC current values (in this case study up to $25 \mathrm{~A}$ ), limiting the equipment's power flow to a set of discrete values, which depend on its voltage. Thus, in theory, this metric could be directly inferred from the EVSE's datasheet, where it is indicated at what voltage the equipment operates. For the considered case study, a $400 \mathrm{~V}$ voltage is reported, which would imply a $400 \mathrm{~W}$ granularity.

From the first component of the test pattern described in Section 2.2, the effective power variations executed by the EVSE can be identified (Figure 4) and isolated in order to provide a distribution of values. For the considered case study, the measured mean AC power output granularity was $350 \pm 38 \mathrm{~W}$ and $416 \pm 14 \mathrm{~W}$ for the discharging and charging operation modes, respectively. Moreover, in Figure 4, it is possible to observe that, for the initial low power set points in the discharging mode, there is a $242.9 \mathrm{~W}$ power flow in the opposite direction to what was requested (i.e., from the grid to the EVSE). This should be interpreted as the EVSE's self-consumption needs (LEDs, ventilation, etc.).

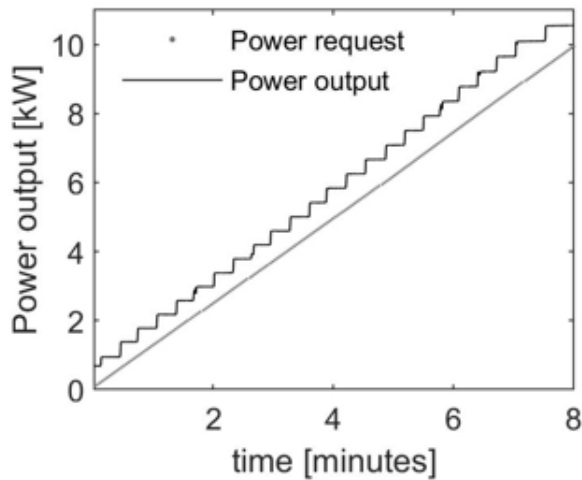

(a)

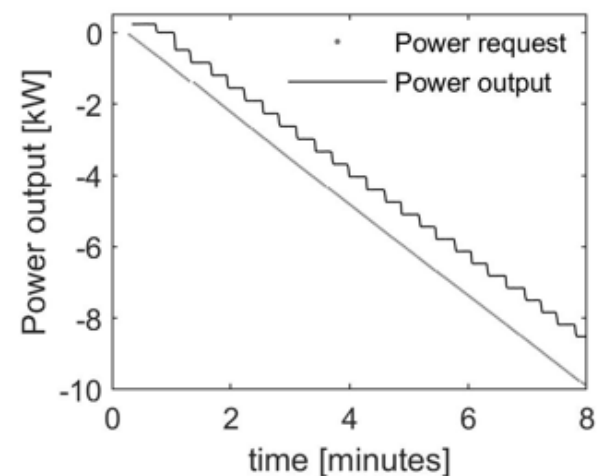

(b)

Figure 4. Comparing the requested power set points (in grey) with the effective AC power output (in black) in (a) charging mode; (b) and discharging mode. The observed steps in power output correspond to the $\mathrm{V} 2 \mathrm{G}$ system granularity. 


\subsection{Response Time}

The response time of a V2G system refers to the time it requires (i.e., its inherent delay) to deliver a requested power set point. As mentioned in the introduction, V2G is better suited for time critical grid services, such as primary reserve (e.g., frequency containment reserve), or secondary reserve. Thus, the response time of a V2G system must be fully understood.

A given power request is only considered to be met when the EVSE output stabilizes after a ramping event. The results allowed us to clearly distinguish between the two steps implied when requesting a change in power output: a communication step, where, as the name implies, the EVSE and EV communicate, with no change in power output; and a ramping step, where the power output effectively varies. This is illustrated in Figure 5.

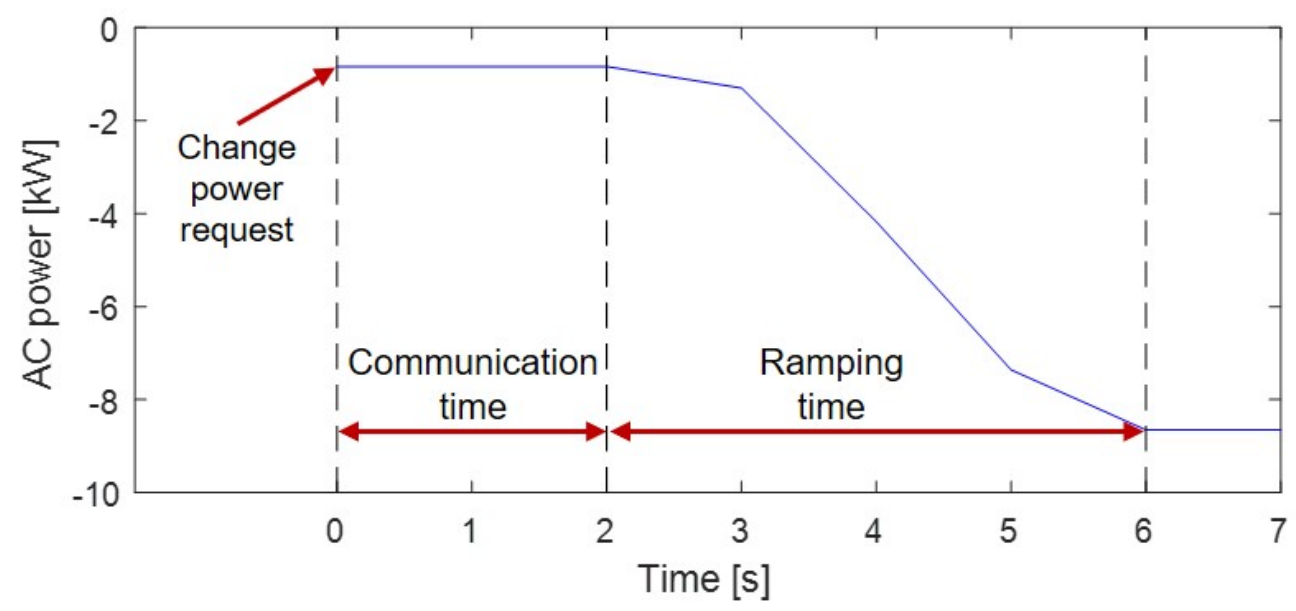

Figure 5. Example of a request for changing the power output from -1 to $-10 \mathrm{~kW}$ (discharge mode). The V2G system takes $6 \mathrm{~s}$ to implement the order (total response time), taking $2 \mathrm{~s}$ to receive the order (communication time) and $4 \mathrm{~s}$ to effectively change the power output (ramping time).

The results showed a considerable dispersion, most likely because the measured times were of the same order of magnitude as the data logger's sampling rate $(1 \mathrm{~Hz})$. This justified the need to repeat this test several times, as averaging the results produced clearer patterns. An alternative would be to use a measuring device with a higher sampling rate.

Both charging and discharging modes achieved similar results. The communication time appeared to be rather stable and independent of the requested power and power ramping, with an average of $2.37 \mathrm{~s}$ and a $0.33 \mathrm{~s}(14 \%)$ standard deviation (Figure 6a). On the other hand, the ramping time appeared to be linearly correlated $\left(\mathrm{R}^{2}=0.95\right)$ with the requested power ramp $(\Delta \mathrm{P})($ Figure $6 \mathrm{~b})$. The EVSE ramping ability was inferred from the inverse of the regression slope as $3.85 \mathrm{~kW} / \mathrm{s}$, with an added 1.67 s constant expense.

The total response time can, thus, be described by a third regression (Equation $(1), \mathrm{R}^{2}=0.81$ ), either directly from the data or by the sum of the regressions of its two components, ranging between 4 and $6.5 \mathrm{~s}$ for the tested $\Delta P$ values.

$$
\text { Total response time }=0.26 \times\left|\Delta P_{\text {request }}\right|+4.04[\mathrm{~s}],
$$

\subsection{Inaccuracy of Response}

The inaccuracy of response of a V2G system indicates how well it corresponds to a given power request in terms of power output. Here, we suggest the use of the mean absolute deviation (MAD) between the requested and effective power output (Equation (2)). 


$$
M A D=\frac{\frac{1}{N} \sum\left|P_{\text {request }}-P_{\text {output }}\right|}{P_{\text {nominal }, \text { EVSE }}},
$$

Considering the system's response time, discussed in the previous section, only measurements made after the ramping process ended and the power flow stabilized should be considered.

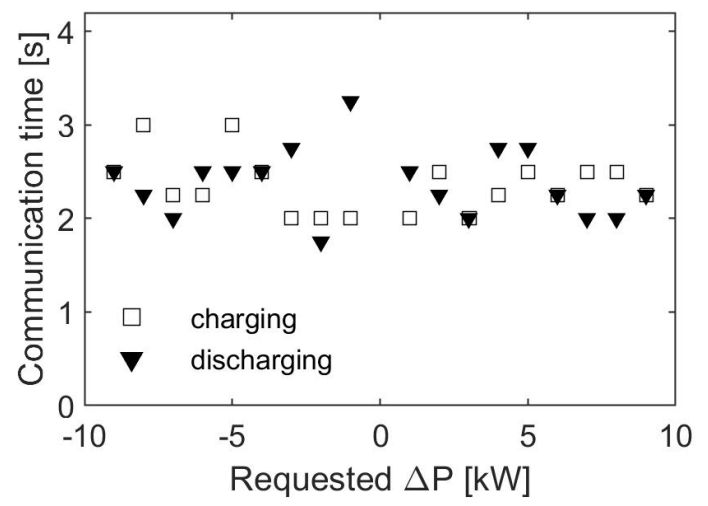

(a)

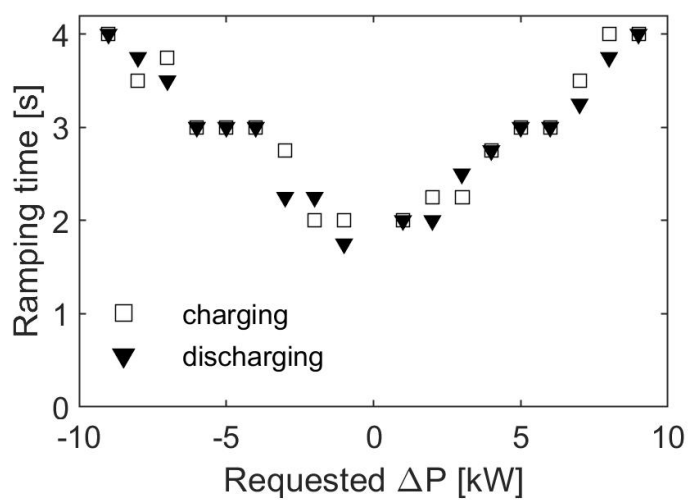

(b)

Figure 6. (a) Communication time; (b) and ramping time as a function of the requested power ramp $(\Delta \mathrm{P})$ for charging (squares) and discharging (filled triangles) modes. Only the ramping time appeared to depend (linearly) on the requested ramping. Each value represents the average of four identical tests, with a standard deviation ranging \pm 0.5 and $\pm 1 \mathrm{~s}$.

Plotting the $\mathrm{AC}$ and DC measurements for the various ramping requests showed that the EVSE implements the requested set points for its DC power flow (Figure 7). Thus, the V2G system is characterized by a $1.8 \%$ and $2.8 \%(180$ and $280 \mathrm{~W})$ DC power MAD for the charging and discharging operation modes, respectively. Figure 7 also shows that the EVSE power saturates when charging at more than $9 \mathrm{~kW}$, with complementary current measurements showing that the current saturated at 23 instead of $25 \mathrm{~A}$. This possibly occurs so that the equipment's AC power does not surpass its nominal value $(10 \mathrm{~kW})$.

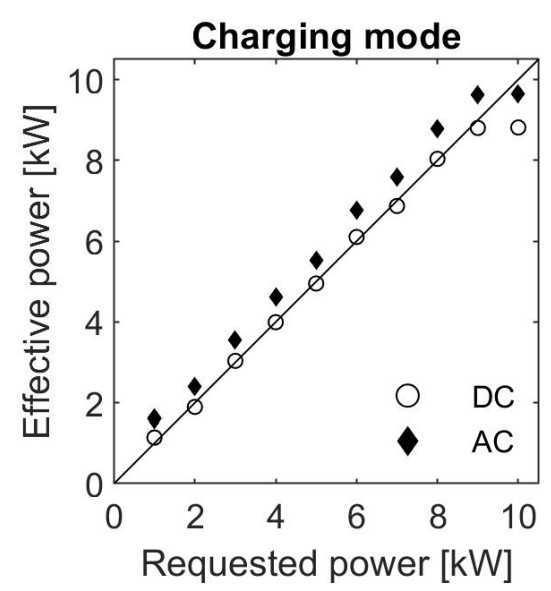

(a)

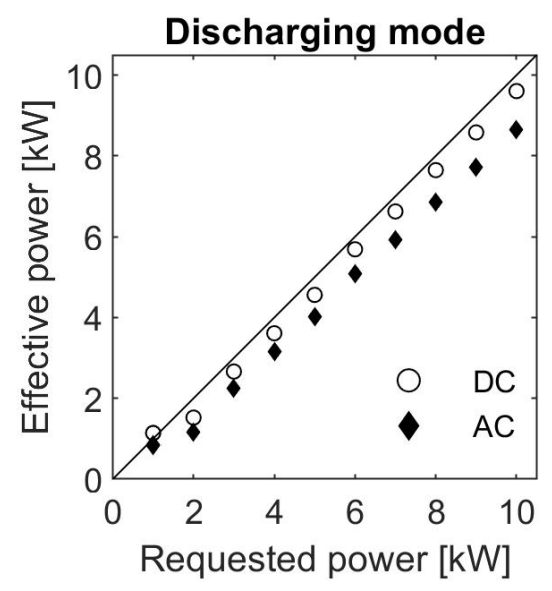

(b)

Figure 7. DC (filled diamonds) and AC (circles) power measurements for the requested set points (a) in charging mode; (b) and in discharging mode. The V2G system is shown to implement the requested power as DC. Each value represents the average of four identical tests, with a standard deviation ranging $\pm 0.01 \mathrm{~kW}$ and $\pm 0.2 \mathrm{~kW}$. 
However, from a grid services provision perspective, it is important to ensure an accurate AC implementation of the V2G system. This can be achieved by describing the AC behavior of the system using linear equations, with the requested power set point as the main input (Equation (3) and Equation (4), both with $R^{2}=0.99$ ).

$$
\begin{gathered}
P_{\text {charge }, A C}=1.011 \times P_{\text {request }}+0.578[\mathrm{~kW}], P_{\text {request }} \in[1 ; 9] \mathrm{kW}, \\
P_{\text {discharge }, A C}=0.926 \times P_{\text {request }}-0.578[\mathrm{~kW}], P_{\text {request }} \in[2 ; 10] \mathrm{kW},
\end{gathered}
$$

Additionally, these equations (as well as their DC counterparts) are of extreme relevance for researchers and $\mathrm{V} 2 \mathrm{G}$ operators, leveraging more accurate and realistic modelling and simulation work.

\subsection{Variability of Response}

It is important to understand how stable the V2G system response is, in order to ensure that it does not introduce any unwanted variability and is effectively viable for imbalance compensation. The metric here described is proposed in [14] and is designated as a precision metric. However, this term can be misleading and can be interpreted as the V2G system accuracy or granularity. Thus, the expression "variability of response" is suggested.

The variability of response can be inferred as the difference between the maximum and minimum power values measured during a given time period in which the power output should be constant. This difference should then be normalized by the EVSE nominal power (Equation (5)).

$$
\text { Variability }=\frac{\max \left(P_{\text {output }, A C}\right)-\min \left(P_{\text {output }, A C}\right)}{P_{E V S E, \text { nominal }}} \times 100[\%],
$$

Because PEVs are better suited for more dynamic (i.e., faster) grid services, the $30 \mathrm{~s}$ period of stable operation considered for each power request (illustrated in Figure 1) was considered to be sufficient for this assessment. Just as with the AC power granularity (Section 3.1), this stability is only of concern for the power grid side and, thus, this analysis is only required for the AC power. The tested V2G system proved to be quite stable, with a variability of response lower than $0.4 \%$ for all tested power set points (e.g., Figure 8 for four tests at $10 \mathrm{~kW}$ output in discharge operation mode).

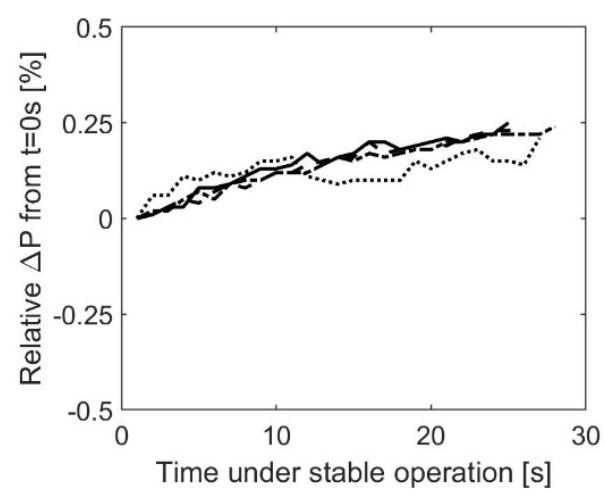

Figure 8. Power output variability for a $10 \mathrm{~kW}$ request in discharging operation mode. At $\mathrm{t}=0 \mathrm{~s}$, the V2G charger meets the requested power set point and is working in stable conditions. The duration of the tests varies slightly, as the $30 \mathrm{~s}$ time period between requests includes the communication (which is slightly variable) and ramping times.

\subsection{EVSE Conversion Efficiency}

The previous metrics are fundamental to fully understand and better implement V2G systems for grid services provision. However, the economic viability of $V 2 G$ is strongly connected to the conversion efficiency of these systems, as it impacts the changes in SOC for the participating PEVs. 
The EVSE conversion efficiency can be determined using Equation (6). The power input $\left(\mathrm{P}_{\text {input }}\right)$ is in AC or DC and the power output ( $\left.P_{\text {output }}\right)$ in DC or AC when operating in charging or discharging modes, respectively. Both charging and discharging modes should be assessed, as each uses different electrical components (a rectifier or an inverter, respectively).

$$
\eta_{\text {charge, discharge }}=\frac{P_{\text {output }}}{P_{\text {input }}}
$$

As expected, conversion efficiency improves as the requested power gets closer to the EVSE nominal value (Figure 9). The calculated values range between $71.4 \%-90 \%$ for the charging mode and $73.4 \%-91.4 \%$ for the discharging mode. These results can be decisive for PEV aggregators when choosing how many PEVs to aggregate and the power request of each one. Aggregating more PEVs can reduce the impact in battery SOC at the individual PEV level (as it would decrease the requested power amount). However, this could be done at the expense of a less efficient operation.

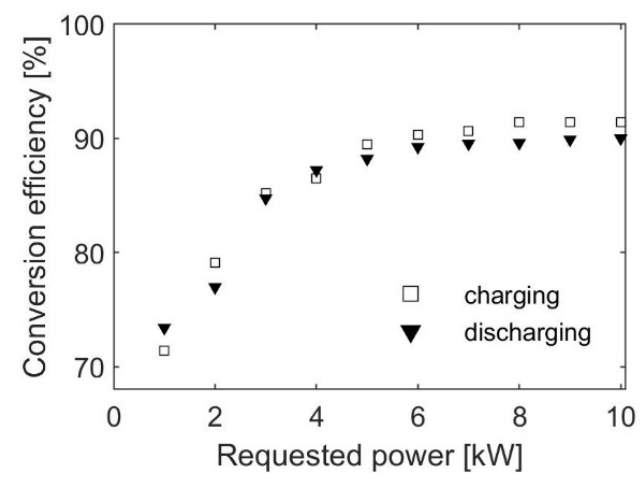

Figure 9. EVSE conversion efficiency for the requested power set points, both in charge (squares) and discharge (filled triangles) modes. The performance degrades for requested power values that fall below $5 \mathrm{~kW}$ ( $50 \%$ of the EVSE nominal power). Each value represents the average of four identical tests, with a standard deviation ranging $\pm 0.2 \%$ and $\pm 0.8 \%$.

\subsection{Reactive Power and Power Factor}

The previously described metrics all focused on active power flow. However, and as described in the introduction, it is not only active power that has a clear impact in power grid operation and efficiency, as PEVs can also play a role in reactive power compensation.

When the tested EVSE is working under normal operating conditions (i.e., with no power factor control imposed by the $\mathrm{V} 2 \mathrm{G}$ operator), reactive power is injected into the power grid in both charging and discharging modes (Figure 10). From a power electronics perspective, and according to the IEC 62053-23 standard [18], the EVSE can be classified as a capacitive load (4th power quadrant) and as an inductive source (3rd power quadrant) when operating in charge and discharge modes, respectively.

From (Figure 10a) it is possible to conclude that when the EVSE is delivering power to the grid (i.e., in discharge mode), the injected reactive power increases as more active power is requested, ranging between 0.62 and $0.75 \mathrm{kVA}_{\mathrm{r}}$; when absorbing power (i.e., charge mode) that injection decreases, ranging between 0.37 and $0.55 \mathrm{kVA}_{\mathrm{r}}$. Nonetheless, the reactive power varies substantially less than the active component; thus, the power factor of the EVSE greatly increases with the requested power output and is close to one when it reaches at least $50 \%$ of its nominal power (Figure 10b). Despite not being represented in the figure, it is important to note that the EVSE injects $1.2 \mathrm{kVA}_{\mathrm{r}}$ into the grid when in "stop mode" (stand-by operation).

Thus, from this kind of assessment, V2G operators and aggregators can plan their operation taking into account the impacts in terms of reactive power. Moreover, it highlights the fact that operating an aggregation of PEVs at lower outputs (below 50\%) is detrimental in terms of the aggregated power factor. 


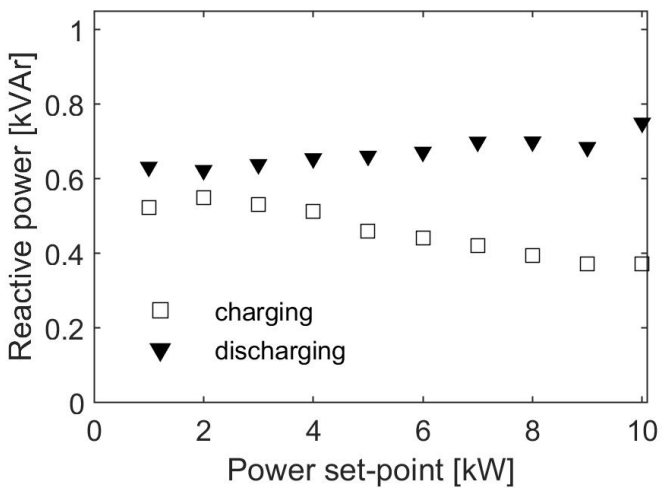

(a)

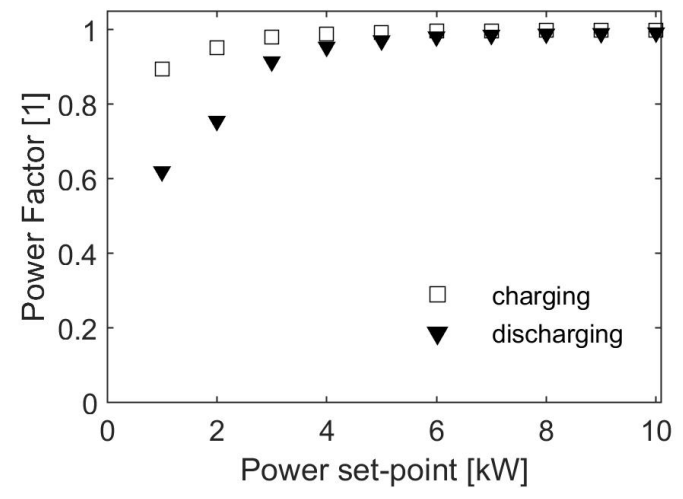

(b)

Figure 10. (a) Reactive power; (b) and power factor for the requested power set points, both in charge (squares) and discharge (filled triangles) modes. In both modes, the EVSE injects reactive power into the grid and the power factor degrades for requested power values that fall below $4 \mathrm{~kW} \mathrm{(40 \%} \mathrm{of} \mathrm{the} \mathrm{EVSE}$ nominal power). Each value represents the average of four identical tests, with a standard deviation ranging \pm 0.3 and $\pm 0.7 \mathrm{kVAr}$ and \pm 0.01 and \pm 0.03 for reactive power and power factor, respectively.

According to the manufacturer's indication, the tested EVSE possesses a control algorithm that allows the V2G operator to define the power factor of the V2G system. To fully assess its performance, the second section of the test pattern proposed in Section 2.2 (i.e., a sequence of power ramping values) was implemented for the requested power factor values from 0 to 1 , in 0.1 steps. A heatmap visualization is suggested to illustrate the effective power factor control as a function of the requested active power output and requested power factor.

The results showed no impact in the actual power factor, independent of the value requested of the EVSE (Figure 11). While these do not fully demonstrate the value of the proposed visualization (i.e., providing a quick understanding of the effective power factor for a given active power and power factor request), the heatmap clearly shows that the control algorithm was not effectively implemented. It is important to stress that the used PEL device was validated for conditions where the effective power factor is known.

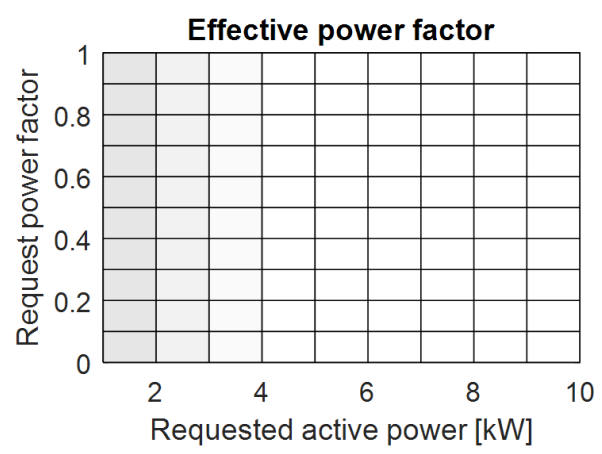

(a)

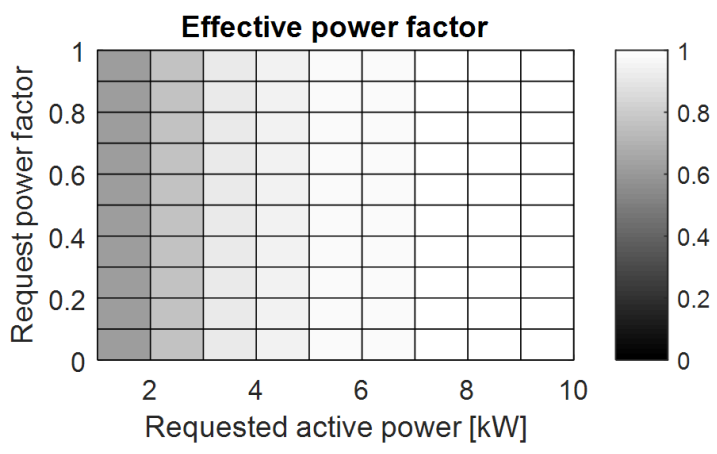

(b)

Figure 11. Effective power factor as a function of the requested active power and the requested power factor (a) in charging operation; (b) and discharging operation. The results show that the power factor control algorithm of the tested V2G charger is, in fact, nonoperational.

\section{Conclusions}

The present work proposes an operational characterization procedure for $\mathrm{V} 2 \mathrm{G}$ systems (i.e., an $\mathrm{BEV}$ connected to a V2G charger) providing grid services. While measuring the power exchanges 
between the V2G system and the power grid, a test pattern is implemented so that the system is characterized for different values of relative power and power ramping levels. Compared to the existing literature, this procedure provides a more in-depth assessment of the system's ramping ability and reactive power implications.

For illustrative purposes, the proposed procedure was implemented on a Nissan Leaf 2015 model connected to a remotely operated commercial V2G fast-charging CHAdeMO charger. Nevertheless, this procedure can be implemented for any kind of charger and plug-in EV, as long as bidirectional power flows are allowed, and the test pattern is implemented for the adequate communication protocol.

Additionally, if only the charging requests are considered, this procedure can also be implemented for dynamic charging-only systems.

Even for dynamic charging systems that do not support bidirectional power flow, the charging component of the test pattern can be implemented. Nonetheless, when considering the EV battery capacity, V2G seems more suitable for BEV.

The suggested procedure is expected to deliver the same results independently of the BEVs capacity (assuming all other characteristics were kept the same). This is justified by the fact that: i) none of the metrics address the battery's capacity or SOC; ii) the proposed procedure has a reduced impact in battery SOC (as its test pattern includes both charging and discharging requests); and iii) battery SoC is not expected to have an impact on V2G performance (other than the vehicle availability to provide services), as Zecchino et al. (2019) showed for the V2G conversion efficiency [14].

Both quantitative results (e.g., inaccuracy and variability of response inferior to $3 \%$ and $0.4 \%$, respectively; communication and ramping times of $2.37 \mathrm{~s}$ and $0.26 \mathrm{~s} / \mathrm{kW}$ ) as well as qualitative results (e.g., conversion efficiency and power factor degradation in power outputs lower than $50 \%$ of the charger's nominal power; the V2G system's implementation of requested power set points as DC power) can be derived from the procedure.

Understanding the operational behavior of these systems enables an optimal operation, maximizing their technical and economic performance. This information can also be of use for V2G regulators to define best practice and benchmarks. For example, these results can be decisive for EV aggregators when choosing the size of the aggregated fleet and the power request of each EV. Aggregating more EVs can reduce the impact in battery SOC at the individual EV level, but will result in a less efficient operation.

As future work, it would be relevant to implement this procedure for different V2G systems, not only to assess how sensitive the performance is depending on the considered charger and/or vehicle, but also to verify how transversal the identified system response (e.g., between efficiency and operating power, or response time and requested ramping) is. Although datasheets from the corresponding EV and EV charger manufacturers can provide insights regarding the performance of the V2G system, the actual V2G system should be tested and characterized as demonstrated by the implemented test procedure of this work. As it would be unpractical to demand that manufacturers test every possible EV-V2G charger combination, V2G operators should characterize their specific systems to obtain better and more accurate information.

Author Contributions: Conceptualization, Â.C.; Methodology, Â.C. and R.A.e.S.; Software, Â.C. and R.A.e.S.; Validation, Â.C., R.A.e.S. and J.S.; Investigation, Â.C.; Resources, Â.C., R.A.e.S. and J.S.; Data curation, Â.C. and R.A.e.S.; Writing-original draft, Â.C.; Writing-review and editing, Â.C., R.A.e.S. and J.S.; Supervision, R.A.e.S. and J.S. All authors have read and agreed to the published version of the manuscript.

Funding: This research was funded by the Portuguese Foundation of Science and Technology (FCT) through the Doctoral grants (PD/BD/114263/2016 and PD/BD/106007/2014) and project UID/GEO/50019/2019 - Instituto Dom Luiz.

Acknowledgments: Financial support from the Portuguese Foundation of Science and Technology (FCT) through the Doctoral grants (PD/BD/114263/2016 and PD/BD/106007/2014) and project UIDB/50019/2020 - IDL is gratefully appreciated. We acknowledge the contributions and fruitful discussions with André Botelho, Pedro M. Ferreira and Marco Pinheiro from EDP Inovação and Miguel C. Brito from Faculdade de Ciências da Universidade de Lisboa.

Conflicts of Interest: The authors declare no conflict of interest. 


\section{References}

1. Habib, S.; Khan, M.M.; Abbas, F.; Sang, L.; Shahid, M.U.; Tang, H. A Comprehensive Study of Implemented International Standards, Technical Challenges, Impacts and Prospects for Electric Vehicles. IEEE Access 2018, 6, 13866-13890. [CrossRef]

2. Das, H.S.; Rahman, M.M.; Li, S.; Tan, C.W. Electric vehicles standards, charging infrastructure, and impact on grid integration: A technological review. Renew. Sustain. Energy Rev. 2020, 120, 109618. [CrossRef]

3. Daina, N.; Sivakumar, A.; Polak, J.W. Electric vehicle charging choices: Modelling and implications for smart charging services. Transp. Res. Part C Emerg. Technol. 2017, 81, 36-56. [CrossRef]

4. Zweistra, M.; Janssen, S.; Geerts, F. Large scale smart charging of electric vehicles in practice. Energies 2020, 13, 298. [CrossRef]

5. Andersen, P.B.; Hashemi, S.; Sousa, T.; Soerensen, T.M.; Noel, L.; Christiensen, B. Cross-brand validation of grid services using V2G-enabled vehicles in the Parker Project. In Proceedings of the 31st International Electric Vehicle Symposium and Exhibition and International Electric Vehicle Technology Conference, Kobe, Japan, 30 September-3 October 2018.

6. Andersen, P.B.; Hashemi, S.; Treholt, C.; Arias, N.B.; Romero, R. Distribution System Services Provided by Electric Vehicles: Recent Status, Challenges, and Future Prospects. IEEE Trans. Intell. Transp. Syst. 2019, 20, 4277-4296.

7. Hu, J.; Morais, H.; Sousa, T.; Lind, M. Electric vehicle fleet management in smart grids: A review of services, optimization and control aspects. Renew. Sustain. Energy Rev. 2016, 56, 1207-1226. [CrossRef]

8. Kempton, W.; Tomić, J. Vehicle-to-grid power fundamentals: Calculating capacity and net revenue. J. Power Sources 2005, 144, 268-279. [CrossRef]

9. Brandt, T.; Wagner, S.; Neumann, D. Evaluating a business model for vehicle-grid integration: Evidence from Germany. Transp. Res. Part D Transp. Environ. 2017, 50, 488-504. [CrossRef]

10. Buja, G.; Bertoluzzo, M.; Fontana, C. Reactive power compensation capabilities of V2G-enabled electric vehicles. IEEE Trans. Power Electron. 2017, 32, 9447-9459. [CrossRef]

11. Sousa, T.; Hashemi, S.; Andersen, P.B. Raising the potential of a local market for the reactive power provision by electric vehicles in distribution grids. IET J. IET Gener. Transm. Distrib. 2019, 13, 2446-2454. [CrossRef]

12. Mouli, G.R.C.; Kaptein, J.; Bauer, P.; Zeman, M. Implementation of dynamic charging and V2G using Chademo and CCS/Combo DC charging standard. In Proceedings of the IEEE Transportation Electrification Conference and Exposition, Busan, Korea, 4 June 2016; pp. 1-6.

13. Martinenas, S.; Marinelli, M.; Andersen, P.B.; Træholt, C. Evaluation of electric vehicle charging controllability for provision of time critical grid services. In Proceedings of the 51st International Universities Power Engineering Conference, Proceedings 2016, Coimbra, Portugal, 6-9 September 2016; pp. 1-5.

14. Zecchino, A.; Thingvad, A.; Andersen, P.B.; Marinelli, M. Test and modelling of commercial V2G CHAdeMO chargers to assess the suitability for grid services. World Electr. Veh. J. 2019, 10, 21. [CrossRef]

15. Chauvin Arnoux. Power Energy Logger PEL 102-103 User's Manual, 10th ed.; Chauvin Arnoux Group: Paris, France, 2017; Available online: https://www.chauvin-arnoux.com/NFPEL100/693780A02_GB.pdf (accessed on 20 February 2020).

16. International Electrotechnical Commission. IEC 61851-1:2017-02. Electric Vehicle Conductive Charging System-Part 1: General Requirements, 3rd ed.; International Electrotechnical Commission: Geneva, Switzerland, 2017.

17. CHAdeMO Association. Available online: http://www.chademo.com/technology/technology-overview/ (accessed on 1 October 2018).

18. International Electrotechnical Commission. IEC 62053-23. Electricity Metering Equipment (a.c.)_Particular Requirements-Part 23: Static Meters for Reactive Energy (classes 2 and 3); International Electrotechnical Commission: Geneva, Switzerland, 2004.

(C) 2020 by the authors. Licensee MDPI, Basel, Switzerland. This article is an open access article distributed under the terms and conditions of the Creative Commons Attribution (CC BY) license (http://creativecommons.org/licenses/by/4.0/). 